\title{
The dual role of the immune system in the course of COVID-19. The fatal impact of the aging immune system
}

\author{
JANUSZ MARCINKIEWICZ ${ }^{1}$, JACEK M. WITKOWSKI ${ }^{2}$, RAFAE OLSZANECKI ${ }^{3}$
}

${ }^{1}$ Chair of Immunology, Faculty of Medicine, Jagiellonian University Medical College, Krakow, Poland

${ }^{2}$ Department of Pathophysiology, Medical University of Gdańsk, Poland

${ }^{3}$ Chair of Pharmacology, Faculty of Medicine, Jagiellonian University Medical College, Krakow, Poland

\begin{abstract}
COVID-19 is a highly contagious respiratory disease caused by the novel coronavirus SARS-CoV-2. Since October 2020 the second wave of the pandemic has been observed around the world, as pathogen specific herd immunity has not been built yet. Moreover, the current, more contagious pathogen carrying the D614G mutation has become the globally dominant form of SARS-CoV-2.

In this article we present the current state of knowledge on the impact of ACE2 and the reninangiotensin system (RAS) and the innate immune system on different outcomes of COVID-19. Especially, we point out the dual role of the immune system and ACE2 in pathogenesis of the disease. Namely, at the initial stage of the infection anti-viral activity of innate immunity is responsible for inhibition of SARS-CoV-2 replication. On the other hand, a dysregulated immune response may cause the detrimental hyperinflammation ("cytokine storm") responsible for the severe course of the disease. Concomitantly, we analyse the roles of ACE2 in both facilitation of infection and abrogation of its effects, as the major cellular entry receptor for SARS-CoV-2 and an important enzyme responsible for tissue protection, respectively. Finally, we discuss the dominant impact of aging on the fatal outcome of COVID-19.
\end{abstract}

Key words: COVID-19, SARS-CoV-2, cytokine storm, inflammaging, angiotensin, ACE2, immunosenescence, interferon.

(Cent Eur J Immunol 2021; 46 (1): 1-9)

\section{Introduction}

The COVID-19 pandemic is a rapidly spreading global outbreak of a novel extremely contagious disease caused by SARS-CoV-2 virus and is much more serious than seasonal influenza. The coronavirus has affected 218 countries and territories with the cumulative number of reported COVID-19 cases over 84 million (> 1,800,000 deaths) worldwide, up to 31 December 2020. In Poland, the current COVID-19 statistics show $~ 1,300,000$ total cases and nearly 30,000 deaths with daily new cases around $400[1,2]$. Concurrently, the number of flu cases and fatal outcomes (deaths) in 2020 (January/April - the main period of flu season in Poland) was the lowest since 2013 and amounted to only 61 deaths [1]. Surprisingly, this positive effect may be related to the coronavirus pandemic. The restrictions resulting from the COVID-19 pandemic and the sanitary regime introduced for this reason in March 2020, which were supposed to stop the spread of coronavirus, also had an impact on the reduction of the flu incidence. Unfortunately, the lowering of restrictions in June-September and irresponsible behaviour of not only coronavirus sceptics, during holidays and various social events, ended up in November with an enormous increased number of new daily cases. Nonetheless, the return of infected and asymptomatic people from holidays to schools and workplaces was the primary factor in the spread of the disease during the spring and autumn outbreaks of COVID-19.

The existing pandemic has triggered enormous mobilization of scientists and clinicians to overcome the disease. Every month thousands of novel sources of information concerning pathogenesis, risk factors and clinical symptoms of COVID-19 are published, but the treatment of patients with the severe form of disease is still not effective $[3,4]$. Importantly, three major clades of SARS-CoV-2 characterized by geographic and genomic specificity can be identified (clades G, V, S) [5]. In particular, clade G, prevalent in Europe, carries a D614G mutation in the spike (S) protein, which is responsible for the initial interaction of the virus with the host human cell [6].

However, it is still unclear whether the distinct case fatality rates (CFR) observed in different countries may be

Correspondence: Prof. Janusz Marcinkiewicz, Chair of Immunology, Faculty of Medicine, Jagiellonian University Medical College, Krakow, Poland, e-mail: mmmarcin@cyf-kr.edu.pl

Submitted: 30.11.2020; Accepted: 2.02.2021 
the consequence of differences in virulence of clades [5]. In our opinion, the outcome of COVID-19 is primarily age-dependent in patients with a similar initial viral load. Namely, in the current year, $23.1 \%$ of the total population in Italy was estimated to be aged 65 years and older, while in India the figure is $6.4 \%$. As expected, CFR in Italy ( 12\%) is much higher than that in India (CFR 2\%) $[1,2]$.

The aim of this paper is to discuss the impact of some risk factors on the severe outcome of COVID-19, especially the factors related to elderly people. We would also like to point out the beneficial and detrimental role of innate immunity in pathogenesis of SARS-CoV-2 infections. We should remember that the entire world population has no adaptive immunity to this disease. COVID-19 is a completely novel human viral infection with no cross-reactivity with former coronavirus diseases, such as SARS-CoV-1 and MERS-CoV [4].

\section{Pathogenesis of COVID-19}

SARS-CoV-2 virus is transmitted from human to human via respiratory droplets. The inhaled virus binds to epithelial cells in the upper airway and starts replicating. Angiotensin converting enzyme 2 (ACE2) is the main receptor for the coronavirus spike glycoprotein $\mathrm{S}[7,8]$. The fate of virus and infected cells depends on the activity of mechanisms of innate immunity. Therefore, in the absence of specific antibodies antiviral defence is related to type I interferons (IFN $\alpha \beta$ ) and NK cells. IFNs are responsible for the reduction of virus replication at the site of infection and activation of NK cells [9, 10]. Effective innate immunity may terminate the course of COVID-19 with full recovery of infected persons. On the other hand, SARSCoV-2 appears to be adapted to evade the host immune response through the suppression of the innate immunity, primarily type I IFNs [11]. The following clinical stages of COVID-19 have been observed: Stage 1 (asymptomatic) - SARS-CoV-2 replicates locally in infected epithelial cells. The patient has no symptoms but is contagious and can spread COVID-19; Stage 2 (mild form) - infection spreads to the upper airways. The patient starts to feel sick with various clinical symptoms. At this stage of the disease symptoms are similar to those observed in flu and common cold; Stage 3 (severe form) - infection spreads to the deepest parts of the lungs, causing severe pneumonia and acute respiratory distress syndrome (ARDS) [12]. Histologically, the hallmark of the early phase of ARDS is diffuse alveolar damage with oedema, haemorrhage and intraalveolar fibrin deposition [13]. Viremia seems not to be the key route of SARS-CoV-2 spreading; however, viral RNA can also be found in peripheral blood and other tissues [14]; Stage 4 systemic multiorgan massive infection including endothelial cells of capillaries as the virus targets and accompanying severe inflammation. Mortality in these patients has been linked to the presence of excessive and uncontrolled pro- duction of proinflammatory cytokines ("cytokine storm") that leads to hyperinflammation, aggravation of ARDS, activation of the coagulation cascade on the damaged pulmonary endothelium and acute respiratory failure [15, 16].

What worsens the situation and impacts on the clinical outcome is that the SARS-CoV-2 infection and in consequence its replication are not limited to the cells of the respiratory system, but may (and does) also involve other cells equipped with the anchoring receptor for the viral S protein - ACE2 and the modifying transmembrane serine protease TMPRSS2, including notably extra-respiratory endothelia, kidneys, liver, heart muscle, neurons and others, potentially leading to multiorgan failure, aggravated by the cytokine storm itself [8]. Importantly, patients may recover after each of the first three stages of COVID-19. However, the disease outcome appears to be age dependent, with young people having mild symptoms or asymptomatic infection, whereas elderly patients tend to have substantial morbidity and mortality [high case fatality rate (CFR)]. For example, in Italy, approximately $90 \%$ of deaths have occurred in COVID- 19 patients aged $\geq 65$ years. It has also been demonstrated that underlying diseases such as diabetes, hypertension and obesity increase the risk of severe COVID-19 outcome [17, 18]. Moreover, patients with pre-existing lung problems such as chronic obstructive pulmonary disease (COPD), lung cancer, cystic fibrosis (CF) and severe asthma are more likely to develop severe symptoms of COVID-19 (Table 1). Surprisingly, the preliminary data collected by the European Cystic Fibrosis Society on the response of CF patients to COVID-19 suggest that the course of the disease may be milder than expected [19, 20].

Most persons with COVID-19 recover and return to normal health. Unfortunately, some patients, especially the elderly with underlying lung diseases and having a wide range of initial symptoms, face an increased risk of "long COVID". The symptoms of "long COVID" (fatigue, shortness of breath, cough, joint pain, headache, "brain fog") can last for weeks or even months after recovery from the acute illness. Apart from the "long COVID" in elderly people, another long-term complication of SARSCoV-2 infection has recently been identified in children. The symptoms of PIMS (PIMS-TS, paediatric inflammatory multisystem syndrome temporally associated with COVID-19) include fever, hyperinflammation, features of Kawasaki disease, toxic shock syndrome or acute abdominal symptoms mimicking appendicitis [2].

\section{Viral infections and defence mechanisms and hyperinflammation/cytokine storm}

During the course of severe COVID-19 two phases of the immune response are observed, the antiviral response (corresponding to asymptomatic and non-severe symptomatic clinical stages 1 and 2) and the inflammatory response (corresponding to severe detrimental respiratory inflammation, clinical stages 3 and 4) [13]. 
Table 1. Major conditions at increased risk of severe COVID-19 illness - in alphabetic order (According to the Centers for Disease Control and Prevention - CDC)

\begin{tabular}{ll}
\hline \multicolumn{1}{c}{ High risk factors } & \multicolumn{1}{c}{ Potential risk factors } \\
\hline Age $>$ 65 years & Asthma (moderate-to-severe) \\
Cancer & Cerebrovascular disease (affects blood vessels and blood supply to the brain) \\
Chronic kidney disease & Cystic fibrosis \\
Chronic obstructive pulmonary disease (COPD) & Hypertension or high blood pressure \\
Heart conditions, such as heart failure, coronary artery & Hyperlipidaemia \\
disease, or cardiomyopathies & Immunocompromised state from blood or bone marrow transplant, immune \\
Immunocompromised state (weakened immune system) & deficiencies \\
from solid organ transplant & Liver disease \\
Obesity & Neurologic conditions, such as dementia \\
Sickle cell disease & Overweight \\
Smoking & Pregnancy \\
Type 2 diabetes mellitus & Sedentary lifestyle \\
& Pulmonary fibrosis (having damaged or scarred lung tissues) \\
& Thalassemia \\
& Type 1 diabetes mellitus \\
& Use of corticosteroids or use of other immune weakening medicines \\
& Vitamin $\mathrm{D}_{3}$ insufficiency \\
\hline
\end{tabular}

\section{Viral response phase}

The viral response phase, common for various respiratory viruses including SARS-CoV-2, is characterised by the type I interferon response. At a site of infection virus-infected epithelial cells and local plasmocytoid dendritic cells produce type I interferons (IFN- $\alpha$, IFN- $\beta$ ) $[9,10]$. The interferon response induces resistance to viral replication in neighbouring cells, increases expression of ligands for receptors on NK cells and activates NK cells to kill virus-infected cells [21]. The early IFN I response may cause rapid viral clearance and patient recovery. In contrast, the delayed and reduced IFN I response results in viral persistence, uncontrolled inflammation and severe disease. Therefore, type I IFNs are key molecules of the defence against SARS-CoV-2 infection at this stage of the COVID-19 pandemic, before achievement of herd immunity. Promisingly, a number of clinical trials on interferon therapy (IFNs $\alpha \beta$ ) in COVID-19 are well underway [2] .

\section{Inflammatory response phase (hyperinflammation, cytokine storm, ARDS)}

A hyperinflammatory immune response may occur if a high viral load stimulates the release of generous amount of reactive oxygen species (ROS) from infected epithelial cells. ROS, in turn, will stimulate the synthesis of NLRP3 and nuclear factor (NF- $\mathrm{kB}$ ), which contribute to development of the cytokine storm [22, 23]. In consequence, neutrophils and macrophages abundantly release cytokines, such as interleukin (IL)-6, tumor necrosis factor $\alpha$ (TNF- $\alpha$ ), IL-1, monocyte chemoattractant protein-1 (MCP-1) and IL-10. Among them, IL-6 and IL-10 were found to be predictive of COVID-19 severity [24]. Importantly, it has been demonstrated that IL-6 plays a primary role in pathogenesis of the cytokine storm [25]. Namely,
IL-6, along with other cytokines, drives an acute phase response that elevates serum ferritin, complement, $\mathrm{C}$ reactive protein (CRP) and pro-coagulant factors. Moreover, as high serum levels of cytokines are inversely related to the total lymphocyte count, low levels of cytotoxic $\mathrm{CD}^{+}$ $\mathrm{T}$ cells may contribute to reduced viral clearance. All these events lead to clinically relevant conditions such as ARDS, sepsis, respiratory failure and potentially even death [26].

\section{SARS-CoV-2 infection - dual role of ACE2}

It is widely recognized that the peptides of the renin-angiotensin system (RAS) acting in an endocrine manner play a crucial role in regulation of blood volume, vascular resistance and electrolyte balance. Moreover, tissue formation of multiple angiotensins via paracrine and autocrine manners plays an important role in local homeostasis of various organs [27]. ACE - the dipeptidyl carboxypeptidase widely distributed in tissues - converts Ang I into Ang II, which by activation of its AT1 receptors is responsible for most pathophysiological effects of RAS. ACE2, an ACE homolog, is broadly expressed throughout the body, reaching high levels in epithelial cells of the nasal mucosa, salivary glands, lungs, kidneys and gastrointestinal tract as well as cardiomyocytes, adipocytes and endothelial cells [28-30]. Thus, the highest ACE2 expression was reported in the heart, kidneys, small intestine, testis, and lungs [31]. Importantly in these tissues, ACE2 represents the main functional counterbalance to ACE as it (1) directly degrades Ang II and (2) is responsible for formation of angiotensin 1-7 [Ang-(1-7)], which by activation of MAS receptors exerts biological activities opposite to Ang II [27, 28, 32, 33]. In 2003 ACE2 was found to be the main cellular virus entry receptor for 
SARS-CoV [34], and recently ACE2 was also established as the main receptor for SARS-CoV-2 [35]. The docking of the receptor binding domain (RBD) of viral envelope spike (S) protein to the peptidase domain of ACE2 represents the initial step of SARS-CoV and SARS-CoV-2 entry to the cell [36]. Similar to SARS-CoV, SARS-CoV-2 utilizes transmembrane serine protease 2 (TMPRSS2), which cleaves $\mathrm{S}$ protein adsorbed to ACE2 and promotes fusion of the viral and cellular membranes. Importantly, TMPRSS2 is highly co-expressed with ACE2 in bronchial and alveolar epithelium. Although ACE2 is probably the most important target for SARS coronaviruses, other membrane-bound proteins have been described as receptors for SARS-CoV-2, e.g. CD209L, CD147/besieging, neuropilin-1 or DPP4/CD26 [36, 37]. Notably, the initial cleavage of the S protein-ACE2 complex triggers further steps of ACE2 proteolysis by TMPRSS2 and membrane protease ADAM17 (a disintegrin and metalloproteinase domain 17), resulting in a loss of ACE2 from the cellular surface by shedding [38]. ACE2 is also directly downregulated upon stimulation of various cells by pro-inflammatory cytokines [39]. It was shown that ACE2 plays an essential role in SARS-CoV dissemination. The lung epithelial cells highly expressing ACE2 have been found to be more prone to viral infection [40]. Moreover, both viral infection and replication as well as acute lung injury are significantly reduced in ACE2-knockout mice [41]. Recent data point to a correlation between the patterns of the tissue virus replication and ACE2 expression [42]; thus, the wide tissue distribution of ACE2 may well explain the broad tissue tropism of the virus. Therefore, it is tempting to speculate that upregulation of ACE2 may lead to higher viral load and poor prognosis, by facilitation of viral entry, yet it should be noted that so far the evidence to support this thesis is not sufficient. Interestingly, plasma ACE2 levels have been reported to be higher in diabetic, cardiac and obese patients [43, 44]. However, the correlation between plasma ACE2 and its tissue levels, as well as their relationships with the poorer outcome of SARS-CoV-2 infection in these patients, remain unclear. Moreover, no age or gender correlations with tissue levels of ACE2 have been found, though it is known that the course of COVID-19 is generally more severe in aged patients and in men. Several lines of evidence, coming mainly from animal models, suggest that the use of drugs such as angiotensin converting enzyme inhibitors (ACEI) and/or angiotensin receptor blockers (ARB) may be associated with increase of the expression of ACE2. However, growing data from animal studies and clinical trials suggest a beneficial effect of these groups of drugs on the course of SARS-CoV-2 infection [45]. Therefore, it is clear that the role of ACE2 in the incidence and course of $\mathrm{CoV}$ infection should be considered in a broader context than that related only to the role of ACE2 in viral entry into cells. It is known that the ACE2/Ang-(1-7)/MAS receptor axis provides the endog- enous counter-balance against ACE/Ang II/AT1 receptor axis overactivation. It prevents the deleterious effects of Ang II at the level of cells (attenuation of reactive oxygen species-related cell death, endothelial protection), tissues (prevention of pathological angiogenesis and fibrosis) and organs (lung kidney and gastrointestinal tract protection, inhibition of thrombosis). This action occurs via two modes (1) ACE2-dependent degradation of Ang II and (2) formation of "beneficial" Ang-(1-7). It has been demonstrated in various models that Ang II exerts multiple proinflammatory actions by direct activation of macrophages to cytokine production and shifting their phenotype toward M1 characteristics [46, 47]. Moreover, Ang II strongly activates endothelium, decreases endothelial production of nitric oxide and hence aggravates neutrophil and monocytes infiltration of affected tissue, increasing the risk of intravascular thrombosis [48]. Considering the downregulation of ACE2 during infection (see above), the prevalence of the Ang II axis over Ang-(1-7) seems to be one of the important factors leading to tissue injury. There are preliminary data indicating that in COVID-19 patients elevated levels of plasma Ang II correlate with the degree of lung injury [49]. Moreover, restoration of proper Ang II/ Ang-(1-7) balance by administration of recombinant ACE2 significantly reverses lung injury in avian influenza lung injury [50], as well as reducing Ang II levels in patients with lung injury caused by RS virus infection [51].

Regarding popular drugs affecting RAS, we know so far that ACEI and ARB present no harm in patients with SARS-CoV-2 infection; however, there are no convincing data indicating whether and in which patients these drugs, presumably increasing ACE2 expression, may bring benefits in COVID-19 [52]. The efficacy of the new strategy - use of recombinant ACE2 in COVID-19 patients - still remains to be established [53]. Nonetheless, it is tempting to speculate that maintaining the balance between the two branches of RAS - ACE/Ang II/AT1 receptor and ACE2/ Ang-(1-7)/MAS receptor - in terms of supporting the latter may be critical for lung protection and better prognosis in patients with SARS-CoV-2 infection. It seems, however, that our approach to the role of ACE2 should be age- and gender-sensitive. SARS-CoV-2 spreads equally among young and elderly persons as well as males and females (which may be in keeping with the lack of significant differences in lung ACE2 expression between these groups), but the mortality risk seems to be higher for males vs. females and elderly vs. young patients [54]. Interestingly, the latter could be partially explained by age- and gender-dependent relationships of lung ACE/ACE2 balance and the immune system in the lungs. A recent study performed on healthy subjects showed the negative correlation of lung ACE2 levels and pulmonary immune signatures (CD8 ${ }^{+}$ cells, NK cells and markers of interferon response) in females and younger persons; in the same study, lung ACE2 levels positively correlated with the pulmonary immune 
signature in males and elderly subjects $[31,55]$. It may suggest that in males and elderly patients with SARSCoV-2 infection high ACE2 levels can surprisingly promote an inflammatory response and lung damage. This interesting hypothesis requires, however, clinical confirmation. ACE2 thus appears to play a doubly complex role in SARS infection: by facilitating the spread of the virus (as a cellular receptor for SARS-CoV-2), and as an important factor limiting tissue damage. The latter action of ACE2 seems to be especially important in female patients and patients at a younger age.

\section{Anti-viral immunity and aging. Immunosenescence and inflammaging}

Aging is associated with profound modifications and composition of the immune system, leading to known symptoms of its dysfunction in elderly people, including: increasing incidence and severity of infectious (both bacterial, and notably viral) diseases and malignancies, autoimmune symptoms, and, generally, lowered responses to vaccines [56-58]. The reasons for these deleterious changes are numerous. They start with the age-associated reduction of output of nadve $\mathrm{T}$ cells from the involuting thymus. This reduction is observable already between the age of 0 and 20 years; there may be a reduction by an order of magnitude between the age of 20 and 50 years, and another order of magnitude between the age 50 and 70 years (making the nadve $\mathrm{T}$ cell output in those 70 years and older 100 times lower than in young adults) $[59,60]$. This greatly reduces the chances for new variants of the $\mathrm{T}$ cell receptors (TCRs) capable of recognizing new epitopes in the elderly, lowering their chances for building a strong adaptive response to new pathogens, e.g. new variants of influenza virus and SARS-CoV-2. Although there exists homeostatic nadve $\mathrm{T}$ cell proliferation in the periphery, it never replaces the missing thymic output in full and likely it is limited to certain variants of $\mathrm{T}$ cell receptors [61]. On the other hand, lifelong re-infections with cognate pathogens, as well as unavoidable development of neoplastic (non-malignant and malignant) cells and challenge with neoantigens, lead to stimulation of those $\mathrm{T}$ cells that have become the clones of memory cells upon the first contact with the antigens. Although they can actively fight cognate pathogens, with time their capacity to do so becomes exhausted, further decreasing the chances for a successful and speedy recovery. These exhausted memory cells do not proliferate vigorously in the response to cognate antigens; this leads to shrinkage or collapse of the TCR repertoire diversity in aged individuals, which essentially precludes their responses to certain pathogens [62]. This phenomenon may also stand behind the reduced responses of elderly people to vaccination, as many of them do not built protective titres of relevant antibodies. This should be brought to the attention of groups trying to develop anti-SARS-CoV-2 vaccines; vaccines destined for older individuals (who may need them more than other age groups) should be formulated differently from those targeting young(er) people in order to ascertain anti-vaccine responses that would be protective against a virulent virus. The anti-shingle vaccine Shingrix and some vaccines against pneumococcal infections which cause the buildup of protective antibody titres in the elderly not differing from that seen in young vaccinated individuals confirm that there is such a possibility. Notably, these vaccines' design contains both increased dosages of pathogen-derived antigens and the specially designed adjuvants that elicit a stronger response from innate immunity, necessary to initiate and maintain robust adaptive responses.

Exhausted immune cells are weaker responders to antigenic stimulation, which itself may hamper the immune response to SARS-CoV-2 by the aging immune systems. However, the next stage in adaptive immunity deterioration is its senescence, called immunosenescence. Immunosenescence can be envisaged as the state in which the adaptive immune cells progressively lose their functional capacities. This is the stage in which more and more T, NK and then also B cells acquire the phenotype of senescent cells, becoming ineffective as immune helpers $\left(\mathrm{CD} 4^{+}\right)$, cytotoxic cells (CD8 ${ }^{+}$and $\mathrm{NK}$ ) or antibody sources (B cells) respectively [63]. Obviously, accumulation of such inefficient lymphocytes results in defective responses to viral and other infections, and to transformed cells $[64,65]$. The molecular background of immunosenescence is first of all deterioration and damage of intracellular signalling (signal transduction) in these immunosenescent cells [66-68].

The senescent cellular phenotype involves enlarged volume, increased activity of an enzyme called the senescence-associated $\beta$ galactosidase (SA- $\beta-\mathrm{Gal}$ ), and short telomeres, and functionally halted proliferation (hence no effector cells) and the senescence-associated secretory phenotype (SASP). SASP converts senescent cells throughout the body (not only those of hematopoietic origin) to efficient producers of proinflammatory cytokines, which are normally manufactured by the immune cells only. Thus, age(ing)-dependent accumulation of senescent cells will increase the inflammatory readiness in the aging organism, even without direct stimulation of the inflammatory process by e.g. pathogenic microorganisms, cancer cells or damaged cells of the individual. This brings us to another side of the "coin" of changes of the immune system associated with aging - inflammaging. Inflammaging (the term was coined by Claudio Franceschi in the last year of the twentieth century) can in the simplest way be defined just as an asymptomatic (or paucisymptomatic) increase in concentration of multiple proinflammatory cytokines (TNF, IL-1, IL-6 etc.). Apparently the source of it may be the challenged cells of the innate immunity as well as the senescent cells with mentioned SASP phenotype $[69,70]$. Unlike at the beginning, when the term started to make its impact on 
our understanding of aging of the immune system and its consequences for human health, inflammaging is now considered not only a deleterious phenomenon leading to (or facilitating) the chronic inflammatory diseases associated with aging, but as a form of overall adaptation of the aging immune system to the decreased functionality of its adaptive part (immunosenescence). Build-up of more vigorous inflammatory response by the innate cells of the elderly may be pro-survival on contact with pathogenic microorganisms and, to some extent, also to its own transformed cells, by facilitating their neutralization and elimination while the adaptive immunity is dwindling [70, 71]. Thus, there is a question to what extent we should intervene to "normalize" immunosenescence and inflammaging. Yes, we should intervene carefully [72], as both inflammaging and immunosenescence are in part not a process of deterioration, but of adaptation [73] to the age-associated changes occurring not only in the immune system itself, but also in the other parts of the aging organism as well.

How does all the above translate to the relation between COVID-19 severity and deadliness and aging? We could say that immunosenescence impairs adaptive responses to SARS-CoV-2, which would lead to the decreased production of both protective antibodies and cytotoxic $\mathrm{CD}^{+}$cells specific for SARS-CoV-2-infected cells. On the other hand, inflammaging, being conductive to increased inflammatory readiness even in the relatively healthy elderly, and clearly participating in the development of chronic inflammatory diseases associated with aging, may participate in the build-up of strong innate immune responses, possibly facilitating and amplifying the hyperinflammation $[74,75]$. Thus, old victims of COVID-19 present not only with a decreased adaptive response, but also with one or more chronic inflammatory comorbidities, which aggravate the course of their disease and increase the risk of fatal outcomes. Interestingly, extremely old persons (centenarians), who are by no means free from chronic inflammatory diseases and cancer, tend to have vigorous inflammatory responses which manifest not only as high proinflammatory cytokine levels, but also high CRP values. Again, their relatively good health and likely, in fact sometimes observed, good survival rate after SARS-CoV-2 infection, seems to be in part the ability to fight the virus itself, but in part also the ability to maintain the balance between pro- and anti-inflammatory mediators, which is typical for these oldest individuals [73, 76], the bearers of a "beneficial genome".

Finally, SARS-CoV-2 infection, as well as infections by the other corona- and dengue viruses, may undergo a phenomenon called antibody enhancement (ADE). In ADE, virus-specific, but non-neutralising antibodies are able to facilitate the enhancement of SARS-CoV-2 entry into target cells via the Fc $\gamma \mathrm{RII}$ receptor and exacerbate the course of COVID-19 [77, 78]. It is currently unknown to what extent ADE may affect the course of COVID-19 in elderly versus young individuals. Certainly further studies of this topic are necessary.

\section{COVID-19: immunoprotection and immunotherapy}

Herd immunity needs to be built to overcome any pandemic and to stop virus transmission. It is achieved when one infected person in a population generates less than one secondary case in the absence of interventions, which corresponds to the effective reproduction number $\mathrm{R}_{\mathrm{e}}$. For SARS-CoV-2, most estimates of $\mathrm{R}_{\mathrm{e}}$ are in the range 2.5-4. Therefore, the herd immunity threshold for SARS-CoV-2 is expected to require $70 \%$ population immunity [79]. Such SARS-CoV-2 specific herd immunity may be achieved either through vaccination or infection with a wild virus. With a vaccine that could protect humans against all clades of SARS-CoV-2, it would be possible to enact a mass worldwide vaccination and end the COVID-19 pandemic. Vaccines generally require years of research and testing before reaching the clinic. However, today, due to enormous mobilization of a great number of scientists, we already have two validated mRNA SARS-CoV-2 vaccines, both safe and very effective [2]. However, the vaccination of millions of adults is needed to achieve herd immunity against COVID-19 in Poland and it will take approximately one year.

In the meantime, passive immunization using high doses of intravenous immunoglobulins (IVIG) is being tested to treat severe and critically ill COVID-19 patients. IVIG, as a blood product containing a mixture of polyclonal immunoglobulin $\mathrm{G}$ ( $\mathrm{IgG}$ ) antibodies, probably suppresses inflammatory reactions by a multifactorial mechanism [80]. On the other hand, for SARS-CoV-2specific immunotherapy, antibodies from convalescent plasma of COVID-19 individuals are purified to prepare the IVIG formulation. This therapy might be effective to treat viral pneumonia as plasma IgGs are able to reach the alveoli through transudation. On the other hand, movement of plasma-derived immunoglobulins into the upper airways has not been described [81]. We hypothesize that protection of these areas of the respiratory tract during early stages of COVID-19 might be achieved by direct application of immunoglobulins via inhalation. The best candidates for such application seem to be nanobodies that bind to the proteins in the SARS-CoV-2 virus. Nanobodies (Nbs) are naturally occurring single-domain heavychain antibodies (without light chain fragments) present in blood of camelids and sharks. They have unique biophysical properties, including small size, that allow inhaled administration of Nbs to prevent adherence of the virus to respiratory epithelial cells [81]. Recently, two high-affinity nanobodies to the spike protein of SARSCoV-2 that block attachment of the spike to ACE2 have been identified and characterised [82]. However, further 
clinical studies are necessary to confirm the therapeutic effectiveness of both plasma-derived immunoglobulins and nanobodies in COVID-19.

\section{Conclusions}

Much remains unknown regarding the epidemiology, pathogenesis, clinical spectrum, and long-term outcomes of COVID-19, "long COVID".

ACE2 appears to play a doubly complex role in SARS infection: by facilitating the spread of the virus (as a cellular receptor for SARS-CoV-2), and as an important factor limiting tissue damage with the strongest effect mainly in female patients and younger patients.

Aging is a major risk factors of the severe COVID-19 outcome. Nevertheless, some underlying diseases (especially chronic inflammatory diseases associated with aging) are high risk factors for such an outcome. Their co-existence with COVID-19 may also put younger individuals at higher risk of an unfavourable outcome of the disease.

In the absence of adaptive immunity, the native immune system plays a primary, but a dual role in the host defence against SARS-CoV-2. The beneficial role: IFN type I reduces virus replication at the early stages of infection. The detrimental role: In severe forms of the disease, IL-6, another cytokine of innate immunity, is a key factor and a driver of the "cytokine storm".

D-dimer rises early, which indicates that coagulopathy acts as a prodrome of the cytokine storm.

The innate immune system is the major, albeit not the only player in inflammaging: the state of proinflammatory readiness, which leads to chronic inflammatory diseases typical for old age and results in the severe outcome of COVID-19.

In patients with mild COVID-19, a rapid decline of specific IgM and IgG titres within 2-4 months has been observed, suggesting that SARS-CoV-2-induced humoral immunity might not be long-lasting in individuals with mild disease.

Importantly, mucosal IgA contributes to early virus neutralization to a greater extent than $\mathrm{IgG}$.

Presently, there is a real hope to overcome the COVID-19 pandemic as the global vaccination has already been launched. However, to achieve herd immunity to SARS-CoV-2 we need to vaccinate a vast majority of adults.

Let us hope that this is the last coronavirus outbreak during which all we have to offer is isolation, quarantine and a sanitary regime, instead of a vaccine and treatment.

\section{Acknowledgments}

We would like to acknowledge support from the Polish Ministry of Science and Higher Education for statutory grants: no. N/41/DBS/000190 (to JM) and no. 02-0058/07/262 (to JMW). We would like to thank Dr Grzegorz Majka for the final editing of the manuscript.

The authors declare no conflict of interest.

\section{References}

1. World Health Organization (2020): WHO coronavirus disease (COVID-19) dashboard. Retrieved from: https://covid19.who. int/.

2. Centers for Disease Control and Prevention (2020): Coronavirus disease 2019 (COVID-19). Retrieved from: https://www. cdc.gov/coronavirus/2019-ncov/index.html.

3. Zhu N, Zhang D, Wang W, et al. (2020): A novel coronavirus from patients with pneumonia in china, 2019. N Engl J Med 382: 727-733

4. Guan WJ, Ni ZY, Hu Y, et al. (2020): Clinical characteristics of coronavirus disease 2019 in china. N Engl J Med 382: 1708-1720.

5. Forster P, Forster L, Renfrew C, Forster M (2020): Phylogenetic network analysis of SARS-CoV-2 genomes. Proc Nat Acad Sci 117: 9241-9243.

6. Ceraolo C, Giorgi FM (2020): Genomic variance of the 2019nCoV coronavirus. J Med Virol 92: 522-528.

7. Lan J, Ge J, Yu J, et al. (2020): Structure of the SARS-CoV-2 spike receptor-binding domain bound to the ACE2 receptor. Nature 581: 215-220.

8. Ou X, Liu Y, Lei X, et al. (2020): Characterization of spike glycoprotein of SARS-CoV-2 on virus entry and its immune cross-reactivity with SARS-CoV-2. Nat Commun 11: 1620.

9. Hadjadj J, Yatim N, Barnabei L, et al. (2020): Impaired type I interferon activity and exacerbated inflammatory responses in severe COVID-19 patients. Science 2020; 369: 718-724.

10. Channappanavar R, Fehr AR, Vijay R, et al. (2016): Dysregulated type I interferon and inflammatory monocyte-macrophage responses cause lethal pneumonia in SARS-CoV-infected mice. Cell Host Microbe 19: 181-193.

11. Kosmaczewska A, Frydecka I (2020): Dysregulation of the immune system as a driver of the critical course of the novel coronavirus disease 2019. Pol Arch Intern Med 130: 779-788.

12. Zhou F, Yu T, Du R, et al. (2020): Clinical course and risk factors for mortality of adult inpatients with COVID-19 in Wuhan, China: a retrospective cohort study. Lancet 395: 1054-1062.

13. Rothan HA, Byrareddy SN (2020): The epidemiology and pathogenesis of coronavirus disease (COVID-19) outbreak. J Autoimmun 109: 102433.

14. Bermejo-Martin JF, González-Rivera M, Almansa R, et al. (2020): Viral RNA load in plasma is associated with critical illness and a dysregulated host response in COVID-19. Crit Care 2020; 24: 691 .

15. Ragab D, Salah Eldin H, Taeimah M, et al. (2020): The COVID-19 cytokine storm; What we know so far. Front Immunol 11: 1446-1446.

16. Sinha P, Matthay MA, Calfee CS (2020): Is a "cytokine storm" relevant to COVID-19? JAMA Intern Med 180: 1152-1154.

17. Guo W, Li M, Dong Y, et al. (2020): Diabetes is a risk factor for the progression and prognosis of COVID-19. Diabetes Metab Res Rev 36: e3319.

18. Sanyaolu A, Okorie C, Marinkovic A, et al. (2020): Comorbidity and its impact on patients with COVID-19. SN Compr Clin Med 2: 1069-1076. 
19. Colombo C, Burgel PR, Gartner S, et al. (2020): Impact of COVID-19 on people with cystic fibrosis. Lancet Resp Med 8: e35-e36.

20. Marcinkiewicz J, Mazurek H, Majka G, Chain B (2020): Are patients with lung cystic fibrosis at increased risk for severe and fatal COVID-19? Interleukin-6 as a predictor of COVID-19 outcome. Pol Arch Intern Med 130: 919-920.

21. Alrubayyi A (2020): NK cells in COVID-19: Protectors or opponents? Nature Rev Immun 20: 520-520.

22. Tang Y, Liu J, Zhang D, et al. (2020): Cytokine storm in COVID-19: The current evidence and treatment strategies. Front Immunol 11: 1708.

23. Gubernatorova EO, Gorshkova EA, Polinova AI, Drutskaya MS (2020): IL-6: Relevance for immunopathology of SARS-CoV-2. Cytokine Growth Factor Rev 53: 13-24.

24. Han H, Ma Q, Li C, et al. (2020): Profiling serum cytokines in COVID-19 patients reveals IL- 6 and IL-10 are disease severity predictors. Emerg Microbes Infect 9: 1123-1130.

25. Tobias Herold M, Arnreich C, Hellmuth JC, et al. (2020): Level of IL-6 predicts respiratory failure in hospitalized symptomatic COVID-19 patients. medRxiv: 2020.04.01.20047381.

26. Wu C, Chen X, Cai Y, et al. (2020): Risk factors associated with acute respiratory distress syndrome and death in patients with coronavirus disease 2019 pneumonia in Wuhan, China. JAMA Intern Med 180: 934-943.

27. Paul M, Mehr AP, Kreutz R (2006): Physiology of local renin-angiotensin systems. Physiol Rev 86: 747-803.

28. Ferrario CM, Ahmad S, Groban L (2020): Twenty years of progress in angiotensin converting enzyme 2 and its link to SARS-CoV-2 disease. Clin Sci 134: 2645-2664.

29. Bujak-Gizycka B, Olszanecki R, Suski M, et al. (2010): Angiotensinogen metabolism in rat aorta: Robust formation of proangiotensin-12. J Physiol Pharmacol 61: 679.

30. Olszanecki R, Madej J, Suski M, et al. (2009): Angiotensin metabolism in rat stomach wall: Prevalence of angiotensin-(1-7) formation. J Physiol Pharmacol 60: 191-196.

31. Li MY, Li L, Zhang Y, Wang XS (2020): Expression of the SARS-CoV-2 cell receptor gene ACE2 in a wide variety of human tissues. Infect Dis Poverty 9: 1-7.

32 Brzozowski T, Ptak-Belowska A, Kwiecien S, et al. (2012): Novel concept in the mechanism of injury and protection of gastric mucosa: Role of renin-angiotensin system and active metabolites of angiotensin. Curr Med Chem 19: 55-62.

33. Skiba DS, Nosalski R, Mikolajczyk TP, et al. (2017): Anti-atherosclerotic effect of the angiotensin 1-7 mimetic AVE0991 is mediated by inhibition of perivascular and plaque inflammation in early atherosclerosis. Br J Pharmacol 174: 4055-4069.

34. Li W, Moore MJ, Vasilieva N, et al. (2003): Angiotensin-converting enzyme 2 is a functional receptor for the SARS coronavirus. Nature 426: 450-454.

35. Letko M, Marzi A, Munster V (2020): Functional assessment of cell entry and receptor usage for SARS-CoV-2 and other lineage B betacoronaviruses. Nat Microbiol 5: 562-569.

36. Shang J, Wan Y, Luo C, et al. (2020): Cell entry mechanisms of SARS-CoV-2. Proc Nat Acad Sci 117: 11727-11734.

37. Daly JL, Simonetti B, Antón-Plágaro C, et al. (2020): Neuropilin-1 is a host factor for SARS-CoV-2 infection. Science 2020; 370: 861-865.

38. Dijkman R, Jebbink MF, Deijs M, et al. (2012): Replication-dependent downregulation of cellular angiotensin-converting enzyme 2 protein expression by human coronavirus NL63. J Gen Virol 93: 1924-1929.
39. De Lang A, Osterhaus ADME, Haagmans BL (2006): Interferon- $\gamma$ and interleukin- 4 downregulate expression of the SARS coronavirus receptor ACE2 in Vero E6 cells. Virology 353: 474-481.

40. Zhang H, Rostami MR, Leopold PL, et al. (2020): Expression of the SARS-CoV-2 ACE2 receptor in the human airway epithelium. Am J Respir Crit Care Med 202: 219-229.

41. Imai Y, Kuba K, Rao S, et al. (2005): Angiotensin-converting enzyme 2 protects from severe acute lung failure. Nature 436 : $112-116$.

42. Harrison AG, Lin T, Wang P (2020): Mechanisms of SARS$\mathrm{CoV}-2$ transmission and pathogenesis. Trends Immunol 41: 1100-1115.

43. Soro-Paavonen A, Gordin D, Forsblom C, et al. (2012): Circulating ACE2 activity is increased in patients with type 1 diabetes and vascular complications. J Hypertens 30: 375-383.

44. Narula S, Yusuf S, Chong M, et al. (2020): Plasma ACE2 and risk of death or cardiometabolic diseases: A case-cohort analysis. Lancet 396: 968-976.

45. Brojakowska A, Narula J, Shimony R, Bander J (2020): Clinical implications of SARS-CoV-2 interaction with renin angiotensin system. J Am Coll Cardiol 75: 3085-3095.

46. Ma L-J, Corsa BA, Zhou J, et al. (2011): Angiotensin type 1 receptor modulates macrophage polarization and renal injury in obesity. Am J Physiol-Renal Physiol 300: F1203-F1213.

47. Dandona P, Dhindsa S, Ghanim H, Chaudhuri A (2007): Angiotensin II and inflammation: The effect of angiotensin-converting enzyme inhibition and angiotensin II receptor blockade. J Hum Hypertens 21: 20-27.

48. Senchenkova EY, Russell J, Esmon CT, Granger DN (2014): Roles of coagulation and fibrinolysis in angiotensin II-enhanced microvascular thrombosis. Microcirculation 21: 401-407.

49. Liu Y, Yang Y, Zhang C, et al. (2020): Clinical and biochemical indexes from 2019-nCoV infected patients linked to viral loads and lung injury. Sci China Life Sci 63: 364-374.

50. Zou Z, Yan Y, Shu Y et al. (2014): Angiotensin-converting enzyme 2 protects from lethal avian influenza A H5N1 infections. Nature Commun 5: 3594.

51. Gu H, Xie Z, Li T, et al. (2016): Angiotensin-converting enzyme 2 inhibits lung injury induced by respiratory syncytial virus. Sci Rep 6: 19840.

52. Kai H, Kai M (2020): Interactions of coronaviruses with ACE2, angiotensin II, and RAS inhibitors - lessons from available evidence and insights into COVID-19. Hypertens Res 43: 648-654.

53. Batlle D, Wysocki J, Satchell K (2020): Soluble angiotensin-converting enzyme 2: A potential approach for coronavirus infection therapy? Clin Sci 134: 543-545.

54. Zhou Y, Zhang Z, Tian J, Xiong S (2020): Risk factors associated with disease progression in a cohort of patients infected with the 2019 novel coronavirus. Ann Palliat Med 9: 428-436.

55. Li G, He X, Zhang L, et al. (2020): Assessing ACE2 expression patterns in lung tissues in the pathogenesis of COVID-19. J Autoimmun 112: 102463.

56. Cohen AA, Kennedy BK, Anglas U, et al. (2020): Lack of consensus on an aging biology paradigm? A global survey reveals an agreement to disagree, and the need for an interdisciplinary framework. Mech Ageing Dev 191: 111316.

57. Fulop T, Larbi A, Khalil A, et al. (2019): Are we ill because we age? Front Physiol 10: 1508-1508.

58. Fulop T, Witkowski JM, Pawelec G, et al. On the immunological theory of aging. In: Aging. Robert L, Fulop T (Eds.). Karger Publishers, Basel, Switzerland 2014; 163-176. 
59. Bains I, Antia R, Callard R, Yates AJ (2009): Quantifying the development of the peripheral naive $\mathrm{CD} 4+\mathrm{t}$-cell pool in humans. Blood 113: 5480-5487.

60. Koetz K, Bryl E, Spickschen K, et al. (2000): T cell homeostasis in patients with rheumatoid arthritis. Proc Nat Acad Sci 97: 9203-9208.

61. Dowling MR, Hodgkin PD (2009): Modelling naive T-cell homeostasis: Consequences of heritable cellular lifespan during ageing. Immunol Cell Biol 87: 445-456.

62. Naylor K, Li G, Vallejo AN, et al. (2005): The influence of age on T cell generation and TCR diversity. J Immunol 174: 7446-7452.

63. Witkowski JM, Larbi A, Le Page A, Fülöp T. Natural killer cells, aging, and vaccination. In: Vaccines for older adults: current practices and future opportunities. Weinberger B (Ed.). Karger Publishers, Basel, Switzerland, 2020; 18-35.

64. Fulop T, Larbi A, Witkowski JM, et al. (2013): Immunosenescence and cancer. Crit Rev Oncog 18: 489-513.

65. Fülöp T, Dupuis G, Witkowski JM, Larbi A (2016): The role of immunosenescence in the development of age-related diseases. Rev Invest Clin 68: 84-91.

66. Fulop T, Le Page A, Fortin C, et al. (2014): Cellular signaling in the aging immune system. Curr Opin Immunol 29: 105-111.

67. Fulop T, Witkowski JM, Le Page A, et al. (2017): Intracellular signalling pathways: Targets to reverse immunosenescence. Clin Exp Immunol 187: 35-43.

68. Le Page A, Dupuis G, Larbi A, et al. (2018): Signal transduction changes in CD4+ and CD8+ T cell subpopulations with aging. Exp Gerontol 105: 128-139.

69. Fulop T, Larbi A, Dupuis G, et al. (2018): Immunosenescence and inflamm-aging as two sides of the same coin: Friends or foes? Front Immunol 8: 1960.

70. Fulop T, Dupuis G, Baehl S, et al. (2016): From inflamm-aging to immune-paralysis: A slippery slope during aging for immune-adaptation. Biogerontology 17: 147-157.

71. Fülöp T, Larbi A, Witkowski JM (2019): Human inflammaging. Gerontology 65: 495-504.

72. Witkowski JM, Bryl E, Fulop T (2019): Should we try to alleviate immunosenescence and inflammaging - why, how and to what extent? Curr Pharm Des 25: 4154-4162.

73. Fulop T, Larbi A, Hirokawa K, et al. (2020): Immunosenescence is both functional/adaptive and dysfunctional/maladaptive. Semin Immunopathol 42: 521-536.

74. Vellas C, Delobel P, De Souto Barreto P, Izopet J (2020): COVID-19, virology and geroscience: A perspective. J Nutr Health Aging 24: 685-691.

75. Bonafč M, Prattichizzo F, Giuliani A, et al. (2020): Inflamm-aging: Why older men are the most susceptible to SARS-CoV-2 complicated outcomes. Cytokine Growth Factor Rev 53: 33-37.

76. Fulop T, Witkowski JM, Olivieri F, Larbi A (2018): The integration of inflammaging in age-related diseases. Semin Immunol 40: 17-35.

77. Wen J, Cheng Y, Ling R, et al. (2020): Antibody-dependent enhancement of coronavirus. Int J Infect Dis 100: 483-489.

78. Lee WS, Wheatley AK, Kent SJ, Dekosky BJ (2020): Antibody-dependent enhancement and SARS-CoV-2 vaccines and therapies. Nat Microbiol 5: 1185-1191.

79. Fontanet A, Cauchemez S (2020): COVID-19 herd immunity: Where are we? Nature Rev Immunol 20: 583-584.

80. Mohtadi N, Ghaysouri A, Shirazi S, et al. (2020): Recovery of severely ill COVID-19 patients by intravenous immunoglobulin (IVIG) treatment: A case series. Virology 548: 1-5.
81. Martinez-Delgado G (2020): Inhaled nanobodies against COVID-19. Nat Rev Immunol 20: 593-593.

82. Huo J, Le Bas A, Ruza RR, et al. (2020): Neutralizing nanobodies bind SARS-CoV-2 spike RBD and block interaction with ACE2. Nat Struct Mol Biol 27: 846-854. 